\title{
Fallopian Tube Obstruction, CTCAE 5.0
}

National Cancer Institute

\section{Source}

National Cancer Institute. Fallopian Tube Obstruction, CT CAE 5.0. NCI Thesaurus. Code C146752.

A disorder characterized by blockage of the normal flow of the contents in the fallopian tube. 